\title{
STUDY ON THE SPECTRAL MIXING MODEL FOR MINERAL PIGMENTS BASED ON DERIVATIVE OF RATIO SPECTROSCOPY-TAKE VERMILION AND STONE YELLOW FOR EXAMPLE
}

\author{
Hengqian Zhao ${ }^{1}$, Yunhao $\mathrm{Hao}^{1}$, Xiaomin $\mathrm{Liu}^{1}$, Miaole Hou ${ }^{2}$, Xuesheng Zhao ${ }^{1}$ \\ ${ }^{1}$ College of Geoscience and Surveying Engineering, China University of Mining and Technology Beijing, Beijing, 100083, China \\ ${ }^{2}$ Beijing University of Civil Engineering and Architecture, Beijing 100044, China
}

KEY WORDS: Chinese Historical Paintings, Mineral Pigments, Spectral Mixing Model, Derivative of Ratio Spectroscopy, Quantitative Analysis

\begin{abstract}
:
Hyperspectral remote sensing is a completely non-invasive technology for measurement of cultural relics, and has been successfully applied in identification and analysis of pigments of Chinese historical paintings. Although the phenomenon of mixing pigments is very usual in Chinese historical paintings, the quantitative analysis of the mixing pigments in the ancient paintings is still unsolved. In this research, we took two typical mineral pigments, vermilion and stone yellow as example, made precisely mixed samples using these two kinds of pigments, and measured their spectra in the laboratory. For the mixing spectra, both fully constrained least square (FCLS) method and derivative of ratio spectroscopy (DRS) were performed. Experimental results showed that the mixing spectra of vermilion and stone yellow had strong nonlinear mixing characteristics, but at some bands linear unmixing could also achieve satisfactory results. DRS using strong linear bands can reach much higher accuracy than that of FCLS using full bands.
\end{abstract}

\section{INTRODUCTION}

Chinese historical heavy-color painting is an important form of Chinese traditional paintings, which has great historical and cultural values. There were many kinds of pigments used in Chinese historical heavy-color paintings, and most of them were mineral pigments(Wu et al., 2014). In addition, vegetal pigments and glues suffer more from oxidative deterioration than mineral pigments(Yan, 2012). Therefore, most of the ingredients preserved in Chinese historical heavy-color painting are mineral pigments. There has always been a great deal of interest in historic artwork with respect to their cultural significance and the physical composition of their materials. Through the analysis of ancient paintings' pigments, the researchers can authenticate the date and origin of cultural relics. It can also provide important reference information for the preservation and restoration of cultural relics by the analysis(Cloutis et al., 2011).

At present, there was few related study on the quantitative analysis of mineral pigments on Chinese ancient paintings based on hyperspectral remote sensing, but had been identified as an important development direction of cultural relic research in China(Tong et al., 2016). The studies of the spectral mixing model of pigments on Western oil paintings started since 2000s. The researchers used spectral unmixing algorithm to solve the contents of different pigment components in the mixture, which provided important reference information for the restoration and historical research of ancient paintings(Balas et al., 2003). However, the Western oil paintings have thick pigment layer, which is mixed with the substrate and attached to the surface of a plate carrier (Dupuis and Menu, 2006). In contrast, Chinese historical heavy-color paintings have much thinner pigment layer, which is integrated with the carrier background (such as Xuan paper), and what spectral mixing model is suitable for them need further study.
Spectral mixing models can be roughly divided into linear mixing model and nonlinear mixing models (Asadzadeh and de Souza Filho, 2016) However, linear mixing model is the most commonly used model, which is simple and has high practical value (Chen et al., 2013). A study showed that the spectra of mineral pigment mixtures were not simple linear mixing results of the endmember pigments (Wang et al., 2005). Besides, the spectral mixing model of minerals varies at different wavelengths. Through selecting the bands suitable for linear mixing modeling, which can be called strong linear bands, and performing linear spectral unmixing on these bands instead of the whole dataset, an extra higher precision for quantitative analysis can be achieved. The derivative of ratio spectroscopy (DRS) is a potential method for the selection of strong linear bands(Zhao et al., 2013b). Previous research indicated that DRS can eliminate the effects of background material in the mixture, and extract the pure spectral information of the target material(Zhao et al., 2013a). Based on the intensity of derivative of ratio spectra at a single wavelength, the content of the target material can be estimated, and the spectral mixing model of different bands can be compared.

Vermillion and stone-yellow are two typical Chinese ancient paintings of mineral pigments, which are often used to mix colors in actual paintings (He, 2008). The two kinds of mineral pigment are selected in this paper as example, make the same particle size of powder has a precise quantitative mixing ratio, strictly control the experimental conditions for getting their mixed spectrum, using fully constrained least squares method based on mixed linear model and derivative of ratio unmixing algorithm to inverting abundances of end elements of mixture pigment, then to evaluate the accuracy and according to the unmixing accuracy of both methods, to carry on the comprehensive analysis to the two mineral pigment spectral mixture model. 


\section{METHODOLOGY}

\subsection{Linear Spectal Mixing Model}

In the linear mixture model, the reflectance of a pixel in each spectral band is expressed as a linear combination of the characteristic reflectances of its component endmembers weighted by their respective areal proportions within the pixel. Thus, the reflectance $r\left(\lambda_{i}\right)$ of a pixel in the $i$ th band is given by

$$
r\left(\lambda_{i}\right)=\sum_{j=1}^{n} F_{j} r_{j}\left(\lambda_{i}\right)+\xi\left(\lambda_{i}\right)
$$

with $i=1,2, \ldots, n$ and $j=1,2, \ldots, m$ where, $r_{j}\left(\lambda_{i}\right)$ denotes the reflectance of the $j$ th component of the pixel in the $i$ th spectral band; $F_{j}$ is the proportion of the $j$ th component in the pixel; $\xi\left(\lambda_{i}\right)$ is the error term in the $i$ th spectral band; $m$ represents the number of spectral bands while $\mathrm{n}$ stands for the number of components in the pixel.

Supposing all the endmembers are included, the following normalization constraint will be satisfied

$$
\sum_{j=1}^{m} F_{j}=1
$$

Moreover, ${ }^{F_{j}}$ should meet the non-negative conditions

$$
F_{j} \geq 0
$$

Given the endmember spectral and the mixed spectral, we can solve the composition of endmembers.

\subsection{Derivative of Ratio Spectroscopy (DRS)}

When a pixel contains only two endmembers $\mathrm{M}$ and $\mathrm{N}$, the Linear Spectral Mixing Model can be simplified as

$$
r(\lambda)=F_{M} \times r_{M}(\lambda)+F_{N} \times r_{N}(\lambda)
$$

If equation (4) is divided by the corresponding equation for the

$$
\frac{r(\lambda)}{r_{N}(\lambda)}=F_{N}+\frac{F_{M} \times r_{M}(\lambda)}{r_{N}(\lambda)}
$$

To obtain the spectral ratio $\frac{r_{M}(\lambda)}{r_{N}(\lambda)}$, the reflectance ratio at each wavelength is calculated. For determining $M$ in the presence of $\mathrm{N}$, use the first derivative of equation (5)

$$
\frac{d}{d \lambda}\left(\frac{r(\lambda)}{r_{N}(\lambda)}\right)=F_{M} \times \frac{d}{d \lambda}\left(\frac{r_{M}(\lambda)}{r_{N}(\lambda)}\right)
$$

Equation (6) indicates that the "derivative ratio spectrum" of the mixture is dependent only on the values of $F_{M}$. If equation (6) is divided by $\frac{d}{d \lambda}\left(\frac{r_{M}(\lambda)}{r_{N}(\lambda)}\right)$ spectrum of $\mathrm{N}$, the following equation can be written:

$$
F_{M}=\frac{\frac{d}{d \lambda}\left(\frac{r(\lambda)}{r_{N}(\lambda)}\right)}{\frac{d}{d \lambda}\left(\frac{r_{M}(\lambda)}{r_{N}(\lambda)}\right)}
$$

At a given wavelength, the fraction of $\mathrm{M}$ can be calculated by

$$
F_{M \lambda_{i}}=\frac{\frac{d}{d \lambda}\left(\frac{r\left(\lambda_{i}\right)}{r_{N}\left(\lambda_{i}\right)}\right)}{\frac{d}{d \lambda}\left(\frac{r_{M}\left(\lambda_{i}\right)}{r_{N}\left(\lambda_{i}\right)}\right)}
$$

$\mathrm{N}$ can be determined by an analogous procedure (wavelength not necessarily the same)

$$
F_{N \lambda_{j}}=\frac{\frac{d}{d \lambda}\left(\frac{r\left(\lambda_{j}\right)}{r_{M}\left(\lambda_{j}\right)}\right)}{\frac{d}{d \lambda}\left(\frac{r_{N}\left(\lambda_{j}\right)}{r_{M}\left(\lambda_{j}\right)}\right)}
$$

For a specific component in the mixture, the abundance RMSE was used for estimation as follows:

$$
R M S E=\sqrt{\frac{\sum_{i=1}^{m}\left(a_{i}-a_{i}^{\prime}\right)^{2}}{m}}
$$

where $a_{i}$ is the real abundance of each mixture, ${ }^{a^{\prime}}{ }_{i}$ is the inversed abundance of each mixture, and $m$ is the number of mixtures.

\section{EXPERIMENTS}

\subsection{Acquisition of experimental data}

The main mineral composition of vermillion is sulfide mercury, while the main mineral composition of stone-yellow is arsenic trisulfide. This experiment chooses the same size of vermillion, stone-yellow pigment powder, firstly measured the density of vermillion and stone-yellow powder, then mixing the two pigment according to seven different ratio of volume(as shown in Table 1). The total volume of pigment is fixed in each group, according to the volume ratio to calculate two kinds of pigment respective volume, according to the density of two kinds of pigment to calculate their own quality, then use micrometer precision electronic balance weigh the pigment powder up. Each group of pigments that had been homogeneous mixing become seven pigment samples, two of which were pure pigment samples and five mixed pigment samples. Seven samples of paint spread on the black paper, keep the black paper smooth, then measured the spectrum of the seven samples of pigments by the SVC HR-1024 portable spectrometer (point spectrometer, FWHM: $\leqq 2.8 \mathrm{~nm}$, $250-1000 \mathrm{~nm} ; \leqq 8.0 \mathrm{~nm}, 1000-1900 \mathrm{~nm} ; \leqq 6.0 \mathrm{~nm}$, 
1900-2500nm), in order to the data quality, the pigment samples are measured twice at least.

\begin{tabular}{|c|c|c|}
\hline Sample No. & Vermilion (V) & Stone-yellow (S) \\
\hline 1 & $0 \%$ & $100 \%$ \\
2 & $10 \%$ & $90 \%$ \\
3 & $30 \%$ & $70 \%$ \\
4 & $50 \%$ & $50 \%$ \\
5 & $70 \%$ & $30 \%$ \\
6 & $90 \%$ & $10 \%$ \\
7 & $100 \%$ & $0 \%$ \\
\hline
\end{tabular}

Table 1 Mixing formula of mineral pigment samples

Deal the original spectral data with overlapping area removal, spectral resampling (make the interval of center wavelength become $1 \mathrm{~nm}$ by resampling form the $350-2500 \mathrm{~nm}$ band), averaging, and some other pretreatment process, using Matlab to drawing images of wavelength and reflectivity, get 7 of reflectance spectra as shown in figure 1, including 2 end elements spectrum and 5 mixed spectrum.

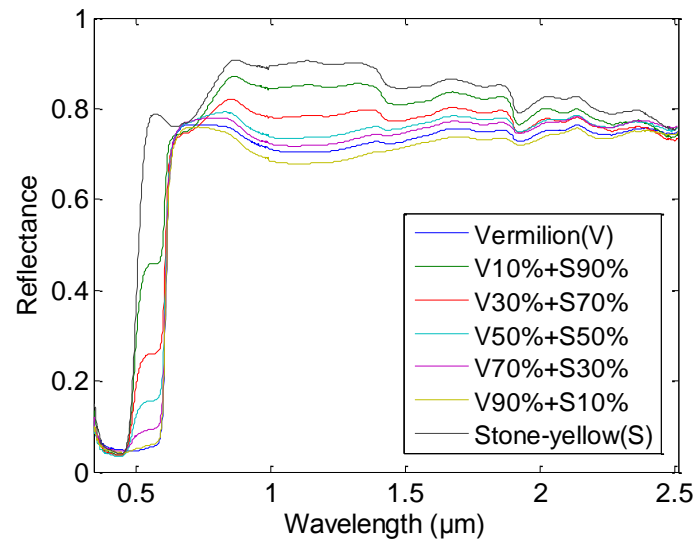

Fig.1 The reflectance spectra of vermilion and stone yellow samples

\subsection{Experimental results}

\subsubsection{Full-band Spectral Mixing Analysis}

Based on the endmember spectra, unmixing of the full-band mixing spectra can be shown by fully constrained least squares method (FCLS), and the abundance of vermillion and stone-yellow in the mixtures can be achieved respectively. The root mean square error (RMSE) between the inverted abundances and actual abundance can be calculated, and the results have been shown in Table 2 .

From the above results, there is great differences between abundances of end elements of vermillion and stone-yellow by the full wave spectrum unmixing inversion and abundance of actual, root mean square error is 0.2828 , unmixing accuracy is low, it shows that the vermillion, stone-yellow mixture mineral pigments do not conform to the linear mixed model in general, nonlinear mixed features performed stronger. This is consistent with the previous analysis of the mineral mixed spectral model (Heylen and Gader, 2014; Singer, 1981).

\subsubsection{Derivative of Ratio Spectroscopy Analysis}

By using the ratio derivative unmixing algorithm, the respective abundance values of vermilion and stone-yellow can be calculated in each band. Taking Sample No. 4 as an example, histogram statistics of vermilion abundance inversion results are shown in Fig.2. It can be seen that the abundances of vermilion obtained from different bands are quite different. The most concentrated solution of the unmixing result lies in the vicinity of $85 \%$, much higher than the true value of $50 \%$. Same as sample No.4, the histograms of the other samples also display apprent deviation between the estimated abundance and the true abundance. This result confirm a conclusion reached in the previous section that the blend of two mineral pigments, vermilion and stone-yellow, does not conform to the linear spectral mixture model as a whole.

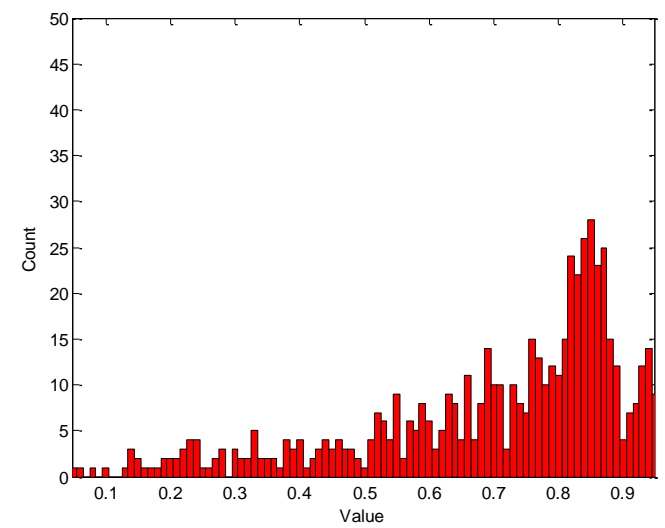

Fig. 2 Histogram of vermilion abundance estimation based on derivative of ratio method (Sample No.4)

Calculate the root mean square error (RMSE) of the endmembers' abundance and the actual abundance of each band inversion, and sort them by the order from low to high to get the 20 band with the highest unmixing accuracy, which is the strong linear band (Table 3). It can be seen that the root mean square error of the strong linear band is less than 0.10 , and the error of many bands is within 0.05 . The accuracy is much higher than that result of the full band spectral unmixing.

\begin{tabular}{|c|c|c|c|c|c|c|c|}
\hline \multirow{2}{*}{ Endmember } & \multirow{2}{*}{ Abundance } & $\begin{array}{c}\text { V10\%+ } \\
\text { S90\% }\end{array}$ & $\begin{array}{c}\text { V30\%+ } \\
\text { S70\% }\end{array}$ & $\begin{array}{c}\text { V50\%+ } \\
\text { S50\% }\end{array}$ & $\begin{array}{c}\text { V70\%+ } \\
\text { S30\% }\end{array}$ & $\begin{array}{c}\text { V90\%+ } \\
\text { S10\% }\end{array}$ & RMSE \\
\hline \multirow{2}{*}{ Vermilion (V) } & Estimated & 0.3852 & 0.6777 & 0.8373 & 0.9282 & 1.0000 & \\
& Actual & 0.1000 & 0.3000 & 0.5000 & 0.7000 & 0.9000 & \\
& Estimated & 0.6148 & 0.3223 & 0.1627 & 0.0718 & 0.0000 & \multirow{2}{*}{0.2828} \\
Stone-yellow(S) & Actual & 0.9000 & 0.7000 & 0.5000 & 0.3000 & 0.1000 & \\
\cline { 1 - 6 } & Difference & 0.2852 & 0.3777 & 0.3373 & 0.2282 & 0.1000 & \\
\hline
\end{tabular}

Table2 Abundance inversion results based on FCLS 
The International Archives of the Photogrammetry, Remote Sensing and Spatial Information Sciences, Volume XLII-3, 2018

ISPRS TC III Mid-term Symposium "Developments, Technologies and Applications in Remote Sensing”, 7-10 May, Beijing, China

\begin{tabular}{|c|c|c|c|c|c|c|c|c|c|c|c|}
\hline & $\lambda / \mathrm{nm}$ & 1104 & 1100 & 1096 & 1093 & 1089 & 365 & 1931 & 1108 & 1085 & 471 \\
& RMSE & 0.0452 & 0.0453 & 0.0530 & 0.0581 & 0.0631 & 0.0632 & 0.0674 & 0.0711 & 0.0719 & 0.0865 \\
& $\lambda / \mathrm{nm}$ & 469 & 472 & 1081 & 468 & 474 & 1077 & 696 & 467 & 697 & 2414 \\
& $\mathrm{RMSE}$ & 0.0887 & 0.0893 & 0.0896 & 0.0920 & 0.0959 & 0.0964 & 0.0966 & 0.0971 & 0.0971 & 0.0999 \\
\hline & $\lambda / \mathrm{nm}$ & 459 & 458 & 461 & 457 & 462 & 1108 & 452 & 455 & 464 & 454 \\
Stone-yellow & $\mathrm{RMSE}$ & 0.0221 & 0.0244 & 0.0255 & 0.0316 & 0.0369 & 0.0385 & 0.0411 & 0.0413 & 0.0432 & 0.0440 \\
& $\lambda / \mathrm{nm}$ & 465 & 467 & 1112 & 1104 & 468 & 451 & 469 & 1931 & 1100 & 471 \\
& $\mathrm{RMSE}$ & 0.0495 & 0.0561 & 0.0574 & 0.0629 & 0.0644 & 0.0657 & 0.0735 & 0.0782 & 0.0828 & 0.0856 \\
\hline
\end{tabular}

Table3 Strong linear bands derived from mixture of vermilion and stone-yellow

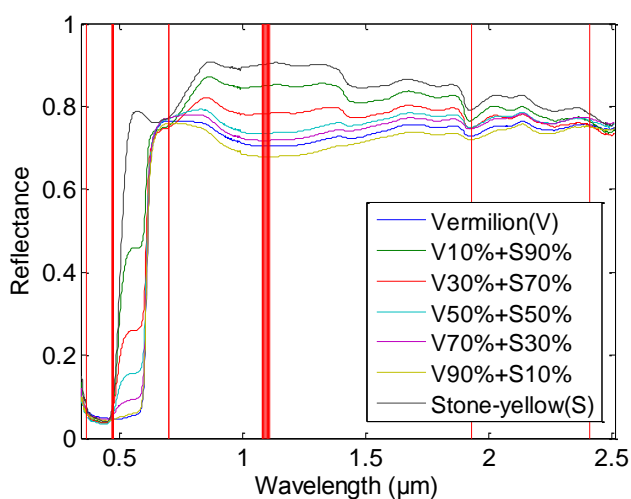

(a)

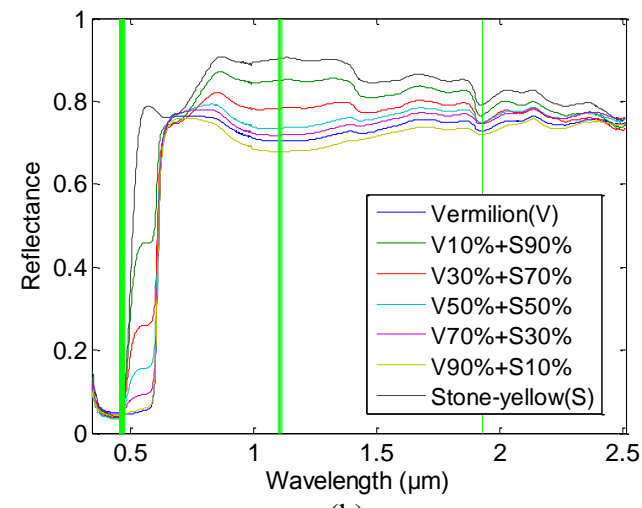

(b)

Fig.3 Strong linear bands on reflectance spectral

(a): strong linear bands of vermilion; (b): strong linear bands of stone yellow

In order to analyze the distribution characteristics of strong linear bands more intuitively, the strong linear bands of vermilion and stone-yellow are respectively marked on the reflectance spectrum, as shown in Fig.3. It can be seen that the strong linear band of the two mineral pigments has a generally equal range of band distribution, which varies locally. This shows that the strong linear band of vermilion and stone-yellow mixed pigments is determined by the spectral characteristics of the two components, but is more affected by the spectral characteristics of the target components locally. This is consistent with the previous study on mineral powder mixtures (Zhao et al., 2013a).

\section{CONCLUSIONS}

In this paper, the mixing spectra of vermilion and stone-yellow powders were analyzed by full-band spectral unmixing and single-band spectral unmixing respectively. The spectral mixture model of Chinese ancient mineral pigments was preliminarily analyzed. The following conclusions were drawn:
(1) The analysis results of full-band spectral mixture model show that the mixtures of vermilion and stone-yellow mineral pigments generally do not fit the linear spectral mixture model and show strong nonlinearity.

(2) The analysis results of single-band spectral mixture model show that the mixtures of vermilion and stone-yellow mineral pigments generally fit the linear spectral mixture model in some bands, show strong linear mixture characteristics.

(3) The range of strong linear band distribution of the two mineral pigments of vermilion and stone-yellow is generally more consistent and different locally. This indicates that the strong linear band is determined by the spectral characteristics of the two mixed components, but is more affected by the spectral characteristics of the target component in the local.

Due to the limited combination of mineral pigments used, further research is required on the combination of different types and different proportions of pigments in ancient Chinese paintings to enhance the applicability of the conclusions. In addition, the next step is to study the non-linear spectral mixture model of mineral pigments and it's spectral unmixing algorithm. With the continuous research, the application of hyperspectral technology in the protection and restoration of ancient Chinese paintings is expected to be further developing.

\section{REFERENCES}

Asadzadeh, S. and de Souza Filho, C.R., 2016. Investigating the capability of WorldView-3 superspectral data for direct hydrocarbon detection. Remote Sensing of Environment, 173: 162-173.

Balas, C. et al., 2003. A novel hyper-spectral imaging apparatus for the non-destructive analysis of objects of artistic and historic value. Journal of Cultural Heritage, 4(1): 330-337.

Chen, J., Richard, C. and Honeine, P., 2013. Nonlinear unmixing of hyperspectral data based on a linear-mixture/nonlinear-fluctuation model. IEEE Transactions on Signal Processing, 61(2): 480-492.

Cloutis, E., Mackay, A., Norman, L. and Goltz, D., 2011. Identification of historic artists' pigments using spectral reflectance and X-ray diffraction properties I. Iron oxide and oxy-hydroxide-rich pigments. Journal of Near Infrared Spectroscopy, 24(1): 27-45.

Dupuis, G. and Menu, M., 2006. Quantitative characterisation of pigment mixtures used in art by fibre-optics diffuse-reflectance spectroscopy. Applied Physics A, 83(4): 469-474.

He Q., 2008. Study of weathering reasons and mechanism on painting and colored drawing of Chinese ancient 
architecture[D]. Northwest University.

Heylen, R. and Gader, P., 2014. Nonlinear spectral unmixing with a linear mixture of intimate mixtures model. IEEE Geoscience and Remote Sensing Letters, 11(7): 1195-1199.

Singer, R.B., 1981. Near - infrared spectral reflectance of mineral mixtures: Systematic Combinations of pyroxenes, olivine, and iron oxides. Journal of Geophysical Research: Solid Earth (1978 - 2012), 86(B9): 7967-7982.

Tong Q., Zhang B. and Zhang L., 2016. Current progress of hyperspectral remote sensing in China[J]. Journal of Remote Sensing, 20(5): 689-707.

Wang, L., Liang, G. and Dang, G., 2005. A study on the protection to relics and the related problems with diffuse reflectance spectroscopy. Spectrochimica Acta Part A Molecular \& Biomolecular Spectroscopy, 61(5): 1021.

Wu F., Yang W. and Li D., 2014. Research on art painting pigment composition recognition based on spectra feature fitting[J], The Journal of Light Scattering, 26(1): 88-92.

Yan L., 2012. Researches on Virtual Recovery of Chinese Paintings Based on Multispectral Technology[D], Tianjin University.

Zhao H., Zhang L., Cen Y., et al., 2013a. Research on the characteristics of strong linearly related bands based on derivative of ratio spectroscopy[J], Journal of Infrared and Millimeter Waves, 32(6): 563-568.

Zhao H., Zhang L., Wu T., et al., 2013b. Research on the model of spectral unmixing for minerals based on derivative of ratio spectroscopy[J], Spectroscopy and Spectral Analysis, 33(1): 172-176. 